\title{
Biotech blockbusters consolidate markets
}

\author{
Stacy Lawrence
}

Biologics are an increasingly significant part of the research pipeline, accounting for more than half of discovery-stage candidates and almost one-fifth of new applications for US Food and Drug Administration

(FDA) approval. Although newly approved HIV treatment Atripla (efa-

\section{Biotech drug candidates by phase}

Large-molecules now comprise $>50 \%$ of discovery-stage candidates and almost $20 \%$ of applications for approval.

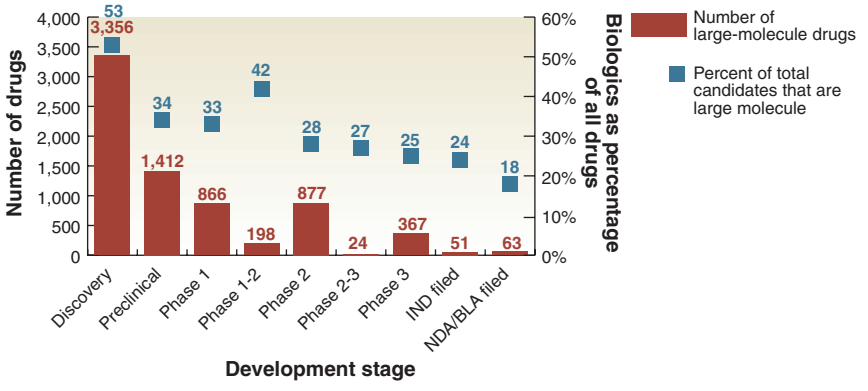

Source: BioPharm Insight

Pending BLA filings

\begin{tabular}{|c|c|c|c|}
\hline Company & Product & $\begin{array}{l}\text { PDUFA date } \\
\text { (BLA) }\end{array}$ & $\begin{array}{c}\text { revenue } 2007 \\
(\$ \text { millions })\end{array}$ \\
\hline $\begin{array}{l}\text { Abbott } \\
\text { Pharmaceuticals }\end{array}$ & $\begin{array}{l}\text { Humira (adalimumab; anti-tumor } \\
\text { necrosis factor alpha) }\end{array}$ & 3/7/07 (sBLA) & 4,031 \\
\hline Acambis/Baxter & $\begin{array}{l}\text { ACAM2000 smallpox vaccine } \\
\text { (clone of Dryvax vaccinia virus derived } \\
\text { in human diploid cell line) }\end{array}$ & 2/18/07 & NA \\
\hline $\begin{array}{l}\text { Alexion } \\
\text { Pharmaceuticals }\end{array}$ & Soliris (eculizumab; anti-C5) & Filed 9/20/06 & 325 \\
\hline Cangene & $\begin{array}{l}\text { Accretropin (generic human growth } \\
\text { hormone) }\end{array}$ & $5 / 17 / 07$ & NA \\
\hline $\begin{array}{l}\text { Dendreon } \\
\text { Pharmaceuticals }\end{array}$ & $\begin{array}{l}\text { Provenge (fusion of prostatic acid } \\
\text { phosphatase to granulocyte-macrophage } \\
\text { colony-stimulating factor) }\end{array}$ & 2007 & NA \\
\hline $\begin{array}{l}\text { Enzon } \\
\text { Pharmaceuticals }\end{array}$ & Onscapar (PEG-L-asparaginase) & Filed 11/05 (sBLA) & 29 \\
\hline Genentech & $\begin{array}{l}\text { Avastin (bevacizumab; anti-vascular } \\
\text { endothelial growth factor) }\end{array}$ & $\begin{array}{l}\text { Filed 5/25/06 and } \\
10 / 11 / 06 \text { (sBLA) }\end{array}$ & 4,775 \\
\hline Genentech & Herceptin (trastuzumab; anti-HER2) & 11/16/06 (sBLA) & 1,700 \\
\hline $\begin{array}{l}\text { GlaxoSmithKline/ID } \\
\text { Biomedical }\end{array}$ & $\begin{array}{l}\text { FluLaval (egg-derived, split virion } \\
\text { influenza vaccine ) }\end{array}$ & $1 / 23 / 07$ & 862 \\
\hline Medlmmune & FluMist (live trivalent influenza virus) & Refiled (sBLA) & 350 \\
\hline Roche Mircera & $\begin{array}{l}\text { CERA (continuous erythropoietin } \\
\text { receptor activator) }\end{array}$ & 2/20/07 & 700 \\
\hline Sanofi-Aventis & $\begin{array}{l}\text { Pentacel (Hemophilus influenzae type } \\
\text { b-diphtheria, tetanus, acellular pertussis } \\
\text { and inactivated poliovirus vaccine) }\end{array}$ & $5 / 25 / 06$ & NA \\
\hline Schering AG & Betaseron (interferon beta-1b) & 12/06 (sBLA) & 1,230 \\
\hline Serono & Rebif (interferon beta- $1 \mathrm{a}$ ) & 2/4/07 (sBLA) & 1,975 \\
\hline $\begin{array}{l}\text { UCB/Nektar } \\
\text { Therapeutics }\end{array}$ & $\begin{array}{l}\text { Cimzia (certolizumab-pegol (Fab' frag- } \\
\text { ment); anti-tumor necrosis factor alpha) }\end{array}$ & $2 / 07$ & 300 \\
\hline
\end{tabular}

BLA, biologic license application; sBLA, supplemental BLA; NA, not available; PDUFA, Prescription Drug User Fee Act. Source: Cowen and Company virenz, emtricitabine, tenofovir), and human papilloma virus vaccine Gardasil are expected to garner $>\$ 1.5$ billion in sales in 2007 , almost all the major \$1-billion-plus projections are for supplemental indications for mainstays like Avastin (bevacizumab) and Herceptin (trastuzumab).

\section{Investigational biotech drugs by indication}

The number of biologic candidates has increased across every major indication, by an average of $>40 \%$

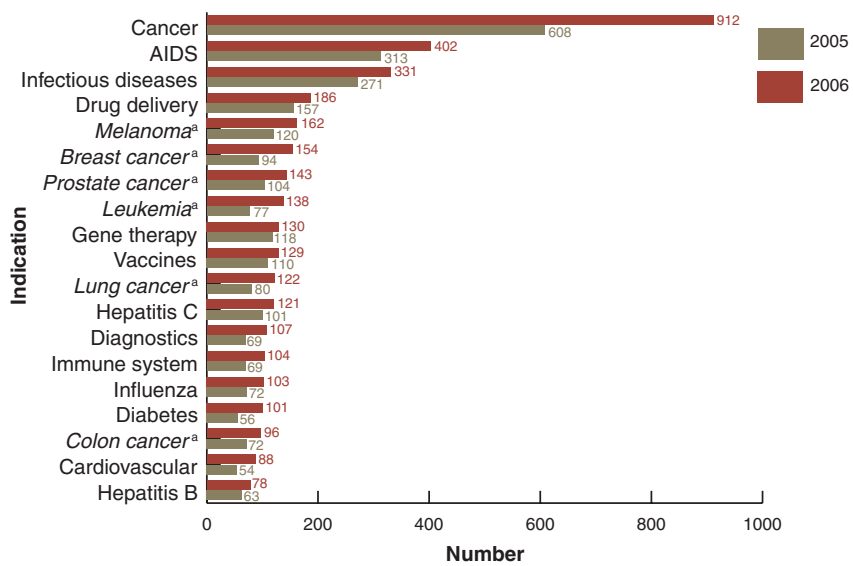

${ }^{a}$ Cancer subtype broken out from total cancer Source: BioPharm Insight

Top ten biotech drugs by global sales

\begin{tabular}{|c|c|c|c|}
\hline Product/company & Type & $\begin{array}{l}2005 \text { sales } \\
\text { (\$ millions) }\end{array}$ & $\begin{array}{c}\text { 1H2006 Sales } \\
\text { (\$ millions) }\end{array}$ \\
\hline $\begin{array}{l}\text { Enbrel (etanercept)/Amgen/ } \\
\text { Wyeth }\end{array}$ & $\begin{array}{l}\text { Recombinant fusion protein; soluble } \\
\text { TNF receptor linked to IgG1 }\end{array}$ & 3,657 & 2,087 \\
\hline $\begin{array}{l}\text { Remicade (infliximab)/ } \\
\text { Centocor }\end{array}$ & Chimeric mAb; anti-TNF-alpha & 3,477 & 2,042 \\
\hline $\begin{array}{l}\text { Aranesp (darbepoetin alfa)/ } \\
\text { Amgen }\end{array}$ & $\begin{array}{l}\text { Recombinant erythropoietin with two } \\
\text { additional N-glycosylation sites }\end{array}$ & 3,273 & 1,948 \\
\hline $\begin{array}{l}\text { Rituxan (rituximab)/ } \\
\text { Biogen-Idec/Genentech }\end{array}$ & Chimeric mAb; anti-CD20 & 3,154 & 1,917 \\
\hline Procrit (erythropoietin)/Amgen & Recombinant erythropoietin & 3,324 & 1,594 \\
\hline $\begin{array}{l}\text { Herceptin (trastuzumab)/ } \\
\text { Genentech }\end{array}$ & Humanized mAb; anti-HER-2 & 1,629 & 1,480 \\
\hline $\begin{array}{l}\text { Neulasta (PEG-filgrastim)/ } \\
\text { Amgen/Dompec Biotech }\end{array}$ & $\begin{array}{l}\text { Recombinant methionyl human } \\
\text { granulocyte colony stimulating factor } \\
\text { (Filgrastim) conjugated to } \\
\text { monomethoxypolyethylene glycol }\end{array}$ & 2,288 & 1,309 \\
\hline Epogen (erythropoietin)/Amgen & Recombinant erythropoietin & 2,455 & 1,217 \\
\hline $\begin{array}{l}\text { Avastin (bevacizumab)/ } \\
\text { Genentech }\end{array}$ & $\begin{array}{l}\text { Humanized } \mathrm{mAb} \text {; anti-vascular } \\
\text { endothelial growth factor }\end{array}$ & 1,264 & 1,134 \\
\hline Epogin/NeoRecormon (Roche) & Recombinant erythropoietin & 1,710 & 898 \\
\hline
\end{tabular}

mAb, monoclonal antibody; TNF, tumor necrosis factor. Source: Signals, Recombinant Capital

Blockbuster biotech approvals

\begin{tabular}{|c|c|c|c|c|c|}
\hline Company/partner & Product & Indication & Status & PDUFA/approval date & Peak potential ( $\$$ billions) \\
\hline Gilead/Bristol & $\begin{array}{l}\text { Atripla (efavirenz, emtricitabine, tenofovir } \\
\text { disoproxil fumarate) }\end{array}$ & HIV & Approved & Approved 7/13/06* & $2.0-2.5$ \\
\hline MedImmune/Merck & $\begin{array}{l}\text { Gardasil (quadrivalent human papillomavirus } \\
\text { types } 6,11,16,18 \text { ) }\end{array}$ & HPV vaccine & Approved & Approved 6/8/06* & 1.50 \\
\hline Genentech/Roche & Avastin (bevacizumab) & Breast cancer & Decision pending (sBLA) & Approvable 9/11/06 & $1.0-1.5$ \\
\hline Genentech/Roche & Avastin (bevacizumab) & Non-small cell lung cancer & Approved (sBLA) & Approved 10/16/06 & $1.0-1.5$ \\
\hline Biogen-Idec/Genentech & Rituxan (rituximab) & ntermediate or aggressive frontline $\mathrm{NHL}$ & Approved (sBLA) & Approved 2/10/06* & $0.5-1.0$ \\
\hline Biogen-Idec/Elan & Tysabri (natalizumab) & Relapsing multiple sclerosis & Approved (sBLA) & Approved 6/5/06** & $0.5-1.0$ \\
\hline Pozen/GlaxoSmithKline & Trexima (sumatriptan succinate and naproxen sodium) & Migraine & Decision pending & Approvable 6/8/06 & $0.5-1.0$ \\
\hline Genentech/Novartis & Lucentis (ranibizumab) & Wet age-related macular degeneration & Approved & Approved 6/30/06* & $0.5-1.0$ \\
\hline Celgene & Revlimid (lenalidomide) & Multiple myeloma & Approved (sNDA) & Approved 6/30/06* & $0.5-1.0$ \\
\hline Amgen & Vectibix (panitumumab) & Refractory colorectal cancer & Approved & Approved 9/27/06 & $0.5-1.0$ \\
\hline
\end{tabular}

*FDA priority review. **Extended PDUFA date. sBLA, supplemental Biologic License Application; sNDA, supplemental New Drug Application; NHL, non-Hodgkins lymphoma; PDUFA, Prescription Drug User Fee Act. Source: Cowen and Company 